Relations industrielles

Industrial Relations

\title{
The Evaluation Interview. By R. A. Fear, New York: McGraw-Hill, 1958.
}

\section{C.-R. Giroux}

Volume 14, numéro 2, avril 1959

URI : https://id.erudit.org/iderudit/1022327ar

DOI : https://doi.org/10.7202/1022327ar

Aller au sommaire du numéro

Éditeur(s)

Département des relations industrielles de l’Université Laval

ISSN

0034-379X (imprimé)

1703-8138 (numérique)

Découvrir la revue

Citer ce compte rendu

Giroux, C.-R. (1959). Compte rendu de [The Evaluation Interview. By R. A. Fear, New York: McGraw-Hill, 1958.] Relations industrielles / Industrial Relations,

14(2), 304-305. https://doi.org/10.7202/1022327ar

Tous droits réservés (C Département des relations industrielles de l’Université Laval, 1959
Ce document est protégé par la loi sur le droit d'auteur. L’utilisation des services d'Érudit (y compris la reproduction) est assujettie à sa politique d'utilisation que vous pouvez consulter en ligne.

https://apropos.erudit.org/fr/usagers/politique-dutilisation/ 


\section{2-L'entrevue d'évaluation mitigée:}

(Tell and Listen Method)

Cette méthode semble plutôt artificielle car elle ne cadre pas beaucoup avec le lien d'autorité qui existe entre patron et employé. Son objectif est de communiquer à l'employé les résultats de l'entrevue et de le laisser réagir par la suite. Dans la première phase, l'interviewer expose les points favorables et défavorables de l'évaluation, dans la deuxième, il explore méticuleusement les réactions et les sentiments de l'employé. Dans ce genre d'entrevue le patron parle encore en juge, mais du moins il permet au subalterne d'exprimer ses sentiments. Il accepte tout comportement hostile ou agressif et n'essaie pas de réfuter les arguments de l'employé. Dans la première phase il est directif, dans la seconde non-directif. Il écoute de façon active et réléchit le contenu émotif des énoncés du subalterne.

Ce genre d'entrevue élimine les situations dans lesquelles le patron est obligé de sauver la face pour protéger son autorité. L'employé épuise son émotivité par catharsis. Il devient objectif et conserve une attitude positive vis-à-vis son patron et son travail.

\section{3-L'entrevue d'évaluation non-directive:}

(The Problem Solving Approach)

Cette méthode n'est pas nouvelle puisqu'elle consiste à appliquer les principes de l'entrevue non-directive de Rogers. Toutefois son utilisation pour l'entrevue d'évaluation est nouvelle. Dans cette entrevue, le patron est un aide, non plus un juge comme dans les deux approches précédentes. Très souvent le but de l'entrevue d'évaluation est d'améliorer l'employé, alors pourquoi manquer l'objectif en communicant l'évaluation? Cet objectif crée un intérêt entre les deux interlocuteurs. Etant moins émotif l'employé décrit plus objectivement les difficultés de son travail. Le patron ne peut pas indiquer les points à discuter, car cela serait un diagnostic et un jugement. Il se limite à stimuler l'initiative de l'employé. En effet l'approche non-directive est caractérisée par la recherche et l'évaluation de diverses solutions à un problème.

Cette méthode d'entrevue est efficace à condition que l'interviewer ait de l'expérience avec la technique non-di- rective. Toutefois, des résultats identiques peuvent être atteints avec l'entrevue mitigée. L'une ou l'autre méthode peut être employée selon l'expérience de l'interviewer et la pensonnalité de l'employé.

Ce volume est un petit chef-d'oeuvre. L'auteur exprime sa pensée de façon claire et concise. Il fallait toute l'expérience de Maier pour saisir et décrire la dynamique humaine qui conditionne le comportement du patron et de l'employé dans l'atmosphère d'une entrevue d'évaluation. Toute personne qui s'intéresse le moindrement aux problèmes inhérents à l'entrevue d'évaluation ne peut ignorer cet ouvrage.

\section{C.-R. Grroux}

The Evaluation Interview. By R. A. Fear, New York: McGraw-Hill, 1958.

Si l'on part du postulat que l'entrevue d'embauchage est la méthode d'administration du personnel la plus utilisée tout en étant l'une des moins efficaces, l'on peut admettre que le présent ouvrage aidera certainement ceux qui s'occupent d'embauchage.

Des recherches ont indiqué que l'entrevue d'embauchage jouissait environ de 5 à $10 \%$ de validité. Pourquoi? Parce que trop souvent l'entrevue n'est pas conduite de façon systématique. Les divers volumes sur l'entrevue d'embauchage sont assez pauvres et ne réussissent pas à éveiller l'intérêt du lecteur. Heureusement ce nouvel ouvrage de Fear éveille un certain intérêt et Il est à date une des meilleures publications sur le sujet.

L'auteur suggère d'attaquer systématiquement quatre facteurs de base: l'histoire \& occupationnelle », l'éducation, l'expérience familiale et l'adaptation sociale. La pensée de l'auteur s'exprime claine et précise dans la plupart des chapitres. Elle donne l'impression que la technique de l'entrevue est facile à maîtriser. Justement, là est le problème, surtout en ce qui concerne linterprétation des faits et la prédiction de $\mathrm{la}$ performance future de l'applicant. Fear décrit des constellations de trats qui devraient solutionner tous les problèmes: introventi, extroverti, agressif, 
intérêt artistique, etc. ... Nous n'hésitons pas à affirmer que la description de chacun d'eux est bien simpliste, beaucoup plus empirique que scientifiquement objective. Considérons le problème suivant: un applicant se montre agressif. Comment pouvons-nous déterminer dans une entrevue même d'une heure si cette agressivité rend l'applicant dynamique et productif ou intolé rant et rigide? Bien des psychologues seraient reconnaissants envers celui qui pourrait leur enseigner comment évaluer de façon précise cet unique trait de personmalité. Pourtant en lisant Fear tout semble si facile.

Pour préparer une entrevue, il faut d'abord que l'interviewer étudie à fond lanalyse de la position concernée, afin d'y découvrir des facteurs spécifiques qui serviront à structurer l'entrevue. Or Fear semble oublier cette phase importante de l'entrevue d'embauchage. Il ne mentionne pas souvent la nécessité de cette phase, s'il la mentionne même. Il aurait pu retrancher du verbiage ici et là et consacrer quelques pages à l'analyse des tâches.

Le contenu du troisième chapitre "How to Become a Good Interviewer" ne correspond pas du tout au titre. L'auteur y rapporte des traits de personnalité propres à la gérance, aux contremaîtres, aux vendeurs, etc. ... Mais M. Fear, comment découvrir les sujets qui deviendront de bons interviewers? Quoi leur enseigner? Quelle expérience doivent-il acquérir? L'auteur aurait pu répondre à ces questions en donnant des références et une bibliographie.

Malgré ces quelques points faibles, ce volume demeure dans l'ensemble intéressant; il vaut la peine d'être lu par tous ceux qui s'intéressent à cette méthode infaillible qu'est l'entrevue d'embauchage. Après avoir été expurgé d'observations psychologiquement naives (page 20), une deuxième édition sera certainement des plus objectives.

\section{C.-R. Ginoux}

Participation in Union Locals. By Arnold S. Tannenbaum \& Robert L. Kahn. Survey Research Center, University of Michigan. Row, Peterson and Company, Evanston, Illinois; White Plains, New York, 1958, 275 pp.
Il y a trente ans, un des premiers observateurs des syndicats ouvriers soulignait la difficulté d'étudier ce genre d'institutions et d'arriver à des généralisations en raison de leur nature complexe, de leur variété et des objectifs divers qu'ils poursuivent. Depuis lors, les sciences humaines ont fait des progrès, les mé.hodes de recherches ont été perfectionnées.

En ces derniers temps, à cause peutêtre de l'importance grandissante du syndicalisme dans notre société industrielle, le nombre d'études scientifiques portant sur le syndicat se multiplie. C'est heureux, car de tels travaux contribuent à mieux faire comprendre ce phénomène social ei surtout à dissiper des jugements hâtifs inspirés souvent par la passion, les préjugés et les intérêts.

Cet ouvrage, Participation in Union Locals, est une excellente contribution à la littérature scientifique sur le syndicalisme. Les auteurs, rattachés au Centre de recherches de l'Université de Michigan, y présentent le fruit d'un travail qu'ils ont effectué sur le problème de la participation dans des syndicats au plan local, ou en d'autres termes, sur la mesure dans laquelle les membres du syndicat se compromettent et consacrent leur énergie à ses activités. Naturellement ils ont été amenés à étendre le champ de leurs observations et à contribuer ainsi à formuler des hypothèses pour expliquer le fonctionnement des syndicats comme institutions. Cette étude enfin leur a permis de vérifier jusqu'à quel point dans l'étude des syndicats on peut utiliser avec succès certaines techniques de recherches.

On essaie de répondre à deux questions. (a) Qu'eniend-on par un membre actif de syndicat et en guoi il diffère d'un membre inactif. (b) Qu'est-ce qu'un syndicat actif et en quoi diffère$t$-il d'un syndicat inactif? Les observations ont été faites auprès de quatre unités locales appartenant à deux unions internationales. On a choisi dans chacune de ces unions internationales un local possédant un haut degré de participation et un autre où l'on croyait ce degré plutôt bas.

Avant d'exposer leur recherche proprement dite, les auteurs ont tenu, dans un chapitre spécial, à expliciter claire- 